\title{
What Shall We Do Next? The Challenges of AI Midway through Its First Century
}

\author{
Paul R. Cohen \\ University of Arizona, USA
}

\begin{abstract}
After half a century of productive work, let us pause to consider what to do next. Looking back we see that Turing's Test was a destination without a map, a goal without a methodology. We see three major, gradual retreats from AI's original goals-general intelligence, knowledge-based intelligence, and problem solving. We see the fragmentation of AI into sub-disciplines and growing uncertainty about who we are and what we want to accomplish. Yet I am optimistic: With an informed understanding of our past we can design and run large-scale, goal-directed research programs-as other organized sciences doand if we do so with vision and discipline we may yet see Turing's Test passedand our understanding of intelligence dramatically increased - before the end of our first century. This is already happening in several areas of AI, as I will illustrate in my talk.
\end{abstract}

